Canadian

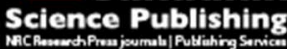

Applied Physiology, Nutrition, and Metabolism Physiologie appliquée, nutrition et métabolisme

\title{
Caffeine Effects on Velocity Selection and Physiological Responses During RPE Production
}

\begin{tabular}{|r|l|}
\hline Journal: & Applied Physiology, Nutrition, and Metabolism \\
\hline Manuscript ID & apnm-2016-0098.R2 \\
\hline Manuscript Type: & Article \\
\hline Date Submitted by the Author: & 02-Jun-2016 \\
\hline Complete List of Authors: & $\begin{array}{l}\text { Green, James; University of North Alabama, HPER } \\
\text { Olenick, Alyssa; Western Kentucky University, School of KRS } \\
\text { Eastep, Caroline; Western Kentucky University, School of KRS } \\
\text { Winchester, Lee; Western Kentucky University, School of KRS }\end{array}$ \\
\hline Keyword: & exercise prescription; perception; training; ergogenic aids; doping \\
\hline & \multicolumn{2}{|l}{} \\
\hline
\end{tabular}


Title: Caffeine Effects on Velocity Selection and Physiological Responses During RPE Production

Submission Type: original investigation

Authors: ${ }^{1,2}$ James M Green, ${ }^{2}$ Alyssa Olenick, ${ }^{2}$ Caroline Eastep, ${ }^{2}$ Lee Winchester

\section{Affiliations:}

${ }^{1}$ Department of Health, Physical Education and Recreation, University of North Alabama, Florence, AL 35632

${ }^{2}$ School of Kinesiology, Recreation and Sport, Western Kentucky University, Bowling Green, KY, 42101

Email addresses of co-authors:

Alyssa Olenick: alyssa.olenick196@topper.wku.edu

Caroline Eastep: caroline.eastep230@topper.wku.edu

Lee Winchester: lee.winchester@wku.edu

Corresponding Author: JM Green

University of North Alabama

Dept. of HPER

1 Harrison Plaza

UNA Box 5073

Florence, AL 35632

Phone: (256) 765-4377

Fax: (256) 765 - 4858

Email:jmgreen@una.edu

Preferred Running Head: Caffeine and Running Velocity Selection 


\begin{abstract}
Purpose: Caffeine blunts estimated Ratings of Perceived Exertion (RPE) but the effects on RPE production are unclear. This study examined effects of acute caffeine ingestion during treadmill exercise where participants exercised at prescribed RPE 4 and 7. Methods: Recreational runners $\left(\mathrm{VO}_{2} \max =51.4 \pm 9.8\right.$ $\left.\mathrm{ml} \cdot \mathrm{kg}^{-1} \cdot \mathrm{min}^{-1}\right)(\mathrm{n}=16)$ completed a maximal treadmill test followed by trials where they selected treadmill velocity (VEL) (1\% grade) to produce RPE 4 and RPE 7 (10 min each). RPE production trials followed caffeine $\left(6 \mathrm{mg} \cdot \mathrm{kg}^{-1}\right)(\mathrm{CAF})$ or placebo (PLA) (counterbalanced) ingestion. Participants were blinded to treadmill velocity but the Omni RPE scale was in full view. Results: Repeated measures ANOVA showed a main effect (trial) for VEL $\left(\mathrm{CAF} \sim 5 \mathrm{~m} \bullet \mathrm{min}^{-1}\right.$ faster $)$ for RPE $4(\mathrm{p}=0.07)$ and RPE $7(\mathrm{p}=0.03)$. Mean HR and $\mathrm{VO}_{2}$ responses were consistently higher for $\mathrm{CAF}$ but failed to reach statistical significance. Individual responses to CAF were labeled positive using a criterion of $13.4 \mathrm{~m} \cdot \mathrm{min}^{-1}$ faster for CAF (vs. PLA). Ten of 32 trials (31\%) were positive responses. In these, systematic increases were observed for HR $\left(\sim 12 \mathrm{~b} \bullet \mathrm{min}^{-1}\right)$ and $\mathrm{VO}_{2}\left(\sim 5.7 \mathrm{ml} \bullet \mathrm{kg}^{-1} \bullet \mathrm{min}^{-1}\right)$. Blunted/stable RER values at higher VEL for positive responders suggest increased FFA reliance during CAF. Conclusions: Mean results show a mild effect of CAF during RPE production. However, individual responses more clearly indicate whether a true effect is possible. Trainers and individuals should consider individual responses to ensure effectively intensity regulation.
\end{abstract}

Key words: exercise prescription; perception; training; ergogenic aids; doping 


\section{Introduction}

Caffeine is noted as an ergogenic aid and the reader is referred reviews focusing on aerobic exercise (Graham 2001) and anaerobic exercise (Davis and Green 2009) for detail. Potential mechanisms of caffeine include reduced muscle fiber recruitment at a given power output (Tallis, et al. 2015) and enhanced free fatty acid (FFA) mobilization with extended time to exhaustion attributed to increased substrate availability (Graham 2001, Costill, et al. 1978). However, effects have been observed during exercise dominated by oxygen-independent (anaerobic) metabolic pathways (Davis and Green 2009) where FFA contributes little to ATP turnover. Enhanced performance in paradigms where FFA is of minimal importance and because of challenges to the Randle effect (Graham 2001) alternate mechanism(s) for ergogenic properties of caffeine must be considered.

Caffeine is proposed to augment pain by competitive blockade of adenosine $A_{1}$ and/or $A_{2}$ receptors (Fredholm, et al. 1999, Sawynok and Yaksh 1993, Zhang 2001), which presents a plausible mechanism for ergogenic properties, particularly during high intensity anaerobic exercise. Similarly, caffeine (vs. placebo) may blunt estimated Ratings of Perceived Exertion (RPE) at set workloads (Birnbaum and Herbst 2004, Killen, et al. 2013) or result in greater work at a similar perceptual rating (Desbrow, et al. 2012, Laurence et al. 2012). Although commonly used for intensity estimation (i.e. "RPE estimation"), RPE may also be used for intensity regulation. Akin to objective methods such as HR, a given RPE (or RPE range) may be prescribed with effort adjusted so feelings of intensity match the prescribed RPE. This 'RPE production' model is a generally accepted approach (Robertson and Noble 1997) and arguably the most convenient model for intensity regulation for daily exercise as it requires no tedious assessments of objective physiological responses such as $\mathrm{HR}, \mathrm{VO}_{2}$ or blood lactate.

Because caffeine reduces estimated RPE (Birnbaum and Herbst 2004, Killen, et al. 2013), caffeine could alter self-selected workload during RPE production. However, this is not well-understood. From our lab, T W Langford, et al. (unpublished observation) showed significantly greater power output selection following caffeine (vs. placebo) ingestion during cycling at prescribed RPE's of $4(\mathrm{CAF}=127 \pm$ $22, \mathrm{PLA}=109 \pm 25$ Watts $)$ and $7(\mathrm{CAF}=161 \pm 36, \mathrm{PLA}=139 \pm 40$ Watts $)$. Corresponding elevations were noted in that study for blood lactate [La] and $\mathrm{VO}_{2}$ as well. Cole et al. (1996) found no significant increase in mean work output during RPE production (RPE: 9, 12, 15) trials from caffeine (vs. placebo), 
but significantly higher accumulated work (CAF: $277.8 \pm 26.1 \mathrm{~kJ}$, PLA: $246.7 \pm 21.5 \mathrm{~kJ}, \mathrm{p}<0.05)$. Also from our lab, Neal, et al. (unpublished observation) found no significant difference for velocity $\left(\mathrm{m} \bullet \mathrm{min}^{-1}\right.$ ) selection at prescribed RPE 4 (CAF: $201.7 \pm 25.8$ vs. PLA: $196.0 \pm 17.5)$ or prescribed RPE 7 (CAF: 236.7 \pm 19.5 vs. PLA $231.8 \pm 21.3$ ) during outdoor (track) running following caffeine (vs. placebo) ingestion. Neal et al. (unpublished observation), Langford et al. (unpublished observation) and Cole et al. (1996) all noted high inter-individual variability among participants in response to caffeine.

If caffeine alters self-regulated intensity, practitioners would need to consider this when using a perceptual model for prescribing exercise. Further, this would have important implications regarding the efficacy of caffeine-containing pre-workout energy drinks. Potential effects of caffeine on intensity regulation using RPE-production is unclear. Therefore, this study examined effects of acute caffeine consumption $\left(6 \mathrm{mg} \cdot \mathrm{kg}^{-1}\right)$ on velocity selection during treadmill exercise at prescribed RPE 4 and 7.

\section{Materials and Methods}

\section{Participants}

Recreationally fit volunteers $(n=16)$ were recruited at two different universities. A power analysis $\left(\right.$ beta $=0.80$, alpha $=0.05$, standard deviation $=20 \mathrm{~m}^{\circ} \mathrm{min}^{-1}$ effect size $\left.13 \mathrm{~m} \bullet \mathrm{min}^{-1}\right)$ showed 16 participants were needed. All procedures were approved by appropriate review boards for the protection of human participants. Prior to data collection, participants signed an informed consent describing requirements. During initial screening, participants completed a Physical Activity Readiness Questionnaire (PAR-Q) (ACSM 2014) and health status questionnaire. Participants were excluded if they had any contradiction to exercise (from PAR-Q), failed to stratify as "low risk" based on known risk factors, were intolerant to lactose (used for placebo) or reported a previous adverse experience with caffeine. Height was assessed to the nearest $\mathrm{cm}$ with a stadiometer (Detecto, Webb City, MO) and weight to the nearest $0.1 \mathrm{~kg}$ with a digital scale (Tanita Corporation, Japan). Body fat percentage was estimated using Lange skin fold calipers (Cambridge, Maryland) and a 3 site method (males: chest, abdomen, and thigh, females: tricep, iliac, thigh) (Pollock, et al. 1980).

$\mathrm{VO}_{2 \max }$ Trial

After descriptive data were collected, participants were instructed to arrive at the lab well-rested (no strenuous activity for $24 \mathrm{~h}$ ), well-hydrated and non-fasted to complete a Bruce treadmill protocol on a 
Trackmaster (Vacumetrics, Ventura, CA) motor driven treadmill to determine maximal oxygen consumption $\left(\mathrm{VO}_{2} \mathrm{max}\right)$. Metabolic data were collected using either a Parvomedics TrueOne 2400 (Parvomedics, Sandy, UT) or Vacumed Vista mini-cps (silver) metabolic system (Vacumetrics, Ventura, CA) with each calibrated according to manufacturer's instructions prior to testing sessions. With the TrueOne, participants donned a nose clip and mouthpiece for collection of expired gas. With the Vacumed, participants were fitted with an appropriately-sized air cushioned mask. Participants wore a Polar heart rate (HR) monitor (Polar, Stamford, CT) for assessment of HR. With the Omni RPE scale (Utter, et al. 2004) in full view, participants estimated overall RPE during the last $10 \mathrm{~s}$ of each min using hand signs. Testing was terminated at volitional exhaustion or if investigators deemed it unsafe to continue. Criteria for achievement of $\mathrm{VO}_{2}$ max included a) plateau of $\mathrm{VO}_{2}$ with increased workload, b) $\mathrm{RER} \geq 1.1$, c) peak RPE 29. All participants achieved 2 of the 3 criteria.

\section{RPE Production Trials}

After a minimum of $24 \mathrm{~h}$ following $\mathrm{VO}_{2}$ max assessment, participants reported to the lab with instructions to be well-rested, well-hydrated (no objective measure of hydration taken) and non-fasted. Participants donned appropriate equipment for collection of expired gas and HR. With the RPE scale in full view and information on the treadmill display concealed, participants warmed-up at a self-selected pace for approximately $2 \mathrm{~min}$. To assimilate outdoor exercise the treadmill was set at 1\% grade throughout (Jones and Doust 1996). Following warm-up, participants used the treadmill keyboard to select a velocity equivalent to an overall RPE 4 (RPE4) on the Omni scale. Gradual adjustments were encouraged and participants tended to achieve the prescribed RPE within a 5 min titration period. Once achieving the prescribed RPE, participants verified they were still at the correct rating 1 min later. At that time, a $10 \mathrm{~min}$ session was initiated with metabolic data recorded at 2, 4, 6, 8 and 10 min. Participants were permitted to adjust velocity at any point necessary to maintain the prescribed RPE. At $10 \mathrm{~min}$ the participant removed the mouthpiece and exited the treadmill for 10 min passive recovery. They then completed the same procedure as above, but adjusted velocity to produce a prescribed RPE 7 (RPE7) with a similar titration period, 1 min verification, and recording of metabolic data at 2, 4, 6, 8, and $10 \mathrm{~min}$ at the prescribed rating.

Production trials were completed $1 \mathrm{~h}$ after participants consumed either caffeine (6 mg kg-1) or matched placebo (maltodextrin) administered in capsule form. Caffeine (CAF) and placebo (PLA) trials 
were counterbalanced to control for ordering and completed approximately the same time of day for each participant. All trials were completed for each participant within a 4 week period. Production of a low (RPE4) intensity always preceded production of the higher (RPE7) intensity. No familiarization trials were employed.

\section{Statistics}

Means and standard deviations were calculated for descriptive variables. To compare velocity selection (VEL), HR $\left(\mathrm{b} \bullet \mathrm{min}^{-1}\right), \mathrm{VO}_{2}\left(\mathrm{ml} \bullet \mathrm{kg}^{-1} \bullet \mathrm{min}^{-1}\right)$ and respiratory exchange ratio (RER) between CAF and PLA, a series of 2 (trial) x 5 (time point) repeated measures ANOVA's were completed using SPSS (v 23.0). Appropriate checks for normality and homogeneity of variance were completed. When appropriate, follow up paired t-tests were used to compare specific time points between trials. In addition to aggregate analyses, individual responses were assessed by identifying an arbitrary difference for VEL between CAF and PLA that reflected a meaningful change. Specific effect sizes were calculated according to Cohen (Cohen 1988).

\section{Results}

Descriptive characteristics of participants were: age (y) $21.1 \pm 2.0$, height $(\mathrm{cm}) 170.0 \pm 8.3$, mass $(\mathrm{kg}) 68.1 \pm 15.1$, percent fat $(\%) 17.2 \pm 7.5, \mathrm{VO}_{2} \max \left(\mathrm{ml} \bullet \mathrm{kg}^{-1} \cdot \mathrm{min}^{-1}\right) 51.4 \pm 9.8$ and peak HR $\left(\mathrm{b} \bullet \mathrm{min}^{-1}\right) 193$ \pm 7 . For RPE4, there was no main effect for time point or trial for VEL $\left(\mathrm{m} \bullet \mathrm{min}^{-1}\right)$, although the difference for trial approached significance ( $p=0.07,1$ tailed test) (Table 1). For RPE4, there was no main effect for $\mathrm{HR}$ but a main effect was observed for time point (Figure 1). The interaction was not significant. For $\mathrm{VO}_{2}$ $\left(\mathrm{ml} \bullet \mathrm{kg}^{-1} \cdot \mathrm{min}^{-1}\right)$ there was no significant main effect for trial but the main effect for time point was significant (Figure 3). There was no significant interaction. For RER there were no significant main effects or interaction (Table 2).

For RPE7, there was a significant main effect for trial for VEL but not for time point and the interaction was not significant (Table 1). There was no significant main effect for HR but the main effect for time point was significant and the interaction was significant (Figure 2). The main effect for $\mathrm{VO}_{2}$ $\left(\mathrm{ml} \cdot \mathrm{kg}^{-1} \cdot \mathrm{min}^{-1}\right)(\mathrm{CAF}=39.3 \pm 7.2, \mathrm{PLA}=37.9 \pm 7.3)$ approached significance $(\mathrm{p}=0.07,1$ tailed test $)$ with a significant main effect for time point but no significant interaction. For RER (CAF $=0.97 \pm 0.01$, PLA $=$ $0.95 \pm 0.01)$ the main effect for trial approached significance $(\mathrm{p}=0.09,1$ tailed test $)$ with a significant main 
effect for time point but no significant interaction (Table 2). For the sample, Cohen's d (mean for CAF mean for PLA divided by standard deviation for PLA) (Cohen 1988) revealed an effect size for VEL of 0.17 for RPE4 and the same for RPE7.

To discern individual responses, $13.4 \mathrm{~m} \bullet \mathrm{min}^{-1}\left(0.5 \mathrm{miles}{ }^{-1}\right)$ was considered a meaningful difference (CAF vs. PLA) in VEL. Using that criterion, participants were identified as positive responders $\left(\geq 13.4 \mathrm{~m} \cdot \mathrm{min}^{-1}\right.$ faster for CAF), non-responders (difference $<13.4 \mathrm{~m} \cdot \mathrm{min}^{-1}$ faster or slower) or negative responders $\left(\geq 13.4 \mathrm{~m} \cdot \mathrm{min}^{-1}\right.$ slower for CAF). For RPE4, the range for the difference in VEL was -18.8 to $+45.6 \mathrm{~m} \cdot \mathrm{min}^{-1}$ and for RPE $7-13.4$ to $+29.5 \mathrm{~m} \cdot \mathrm{min}^{-1}$. For RPE4, there were $6(37.5 \%)$ positive responders, $8(50 \%)$ non-responders and $2(12.5 \%)$ negative responders. For RPE7, there were 4 (25\%) positive responders, $11(68.8 \%)$ non-responders and $1(6.3 \%)$ negative responder.

\section{Discussion}

Potential mechanisms associated with ergogenic properties of caffeine include reduced fiber recruitment at a given intensity (Tallis et al. 2015), augmented pain (Graham 2001, Davis and Green 2009) or enhanced substrate (FFA) availability though the latter particularly is tenuous (Graham 2001). RPE estimations may be blunted (Birnbaum and Herbst 2004, Killen et al. 2013), but effects on RPE production are unclear. This study examined the influence of caffeine (6 on velocity selection (VEL) and corresponding physiological responses during treadmill exercise. Results show a mild overall faster VEL for CAF (vs. PLA). Responses among participants varied widely necessitating evaluation beyond aggregate results. In those who benefited, $\mathrm{HR}$ and $\mathrm{VO}_{2}$ were systematically elevated with higher VEL. Although evidence supporting caffeine-induced increased FFA is equivocal, RER in the current study suggests a potential impact though the magnitude is difficult to discern. Key novel findings are that caffeine may alter perceptually-regulated intensity regulation, though effects must be considered per individual.

Responders vs. Non-responders

Current results show an overall faster mean VEL from acute caffeine ingestion with the observation consistent for a lower (RPE4) and higher (RPE7) prescribed intensity (Table 1). The magnitude

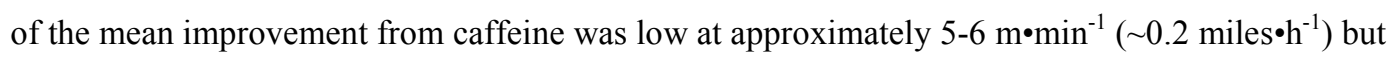
consistent at specific time points (Table 1), though some $\mathrm{p}$ values were marginal. These outcomes are explained primarily by two observations. First, large standard deviations for VEL reflect a sample of 
runners highly diverse in training pace. This would be expected based on wide variability for $\mathrm{VO}_{2} \max$ (Table 1). With a large standard deviation, a larger effect is required to attain statistical significance. Certainly, with a more homogenous group of runners with lower variability in skill (i.e. cross country runners) smaller differences would be statistically more meaningful. However, sampling from a cross country team precludes inference to the general population of recreational runners, the intended focus of the current investigation.

A second factor is the variation among participants with regards to treatment (caffeine) response. With ergogenic aids, percentages of participants may respond favorably, unfavorably or not at all. In that paradigm, inferring conclusions to an entire population from aggregate analyses of the entire sample is tenuous. If a true effect in some is diluted by lack of (or negative) effect in others, then a universal conclusion of no effect is inaccurate, potentially in a considerable portion of individuals. A review of literature on the influence of caffeine on perceptual responses to exercise would show equivocal findings and often small magnitude of change in studies observing an overall benefit. We propose this is potentially linked to diverse responses among individuals and a need to analyze beyond aggregate analyses. While current aggregate analyses indicate significance for mean results, the magnitudes for mean differences (CAF vs. PLA) are small (Table 1). However, evaluating individual responses presents a more thorough assessment and more clearly indicates a true effect occurred in a portion of participants while others experienced no effect or a negative effect. As indicated in Results, we employed a criterion of $13.4 \mathrm{~m} \bullet \mathrm{min}^{-1}$ $\left(0.5 \mathrm{~m} \bullet \mathrm{hr}^{-1}\right)$ to categorize individual responses. See Results for number of responders per intensity. Collectively, of 32 trials, 10 (31\%) improved, 19 (59\%) incurred minimal change and 3 (9\%) responded unfavorably. Neal et al. (unpublished observation) showed no significant effect of caffeine on mean velocity selection for RPE4 (CAF: $201.7 \pm 25.8$ vs. PLA: $196.0 \pm 17.5)$ or RPE7 (CAF: $236.7 \pm 19.5$ vs. PLA $231.8 \pm 21.3$ ) during outdoor running. However, the magnitude of change for CAF was similar to that observed in the current study ( $\sim 5-6 \mathrm{~m} \bullet \mathrm{min}^{-1}$ faster for CAF). In that study, 9 of 20 total trials $(46 \%)$ were

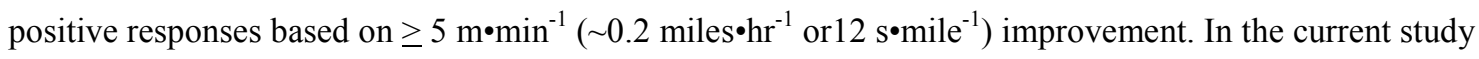
we employed a comparatively more conservative criterion for positive responses $\left(13.4 \mathrm{~m} \cdot \mathrm{min}^{-1}\right)$. This was warranted as Neal et al. tested higher fit (all male) runners $\left(\mathrm{VO}_{2} \max =60.8 \pm 3.9 \mathrm{ml} \cdot \mathrm{kg}^{-1} \cdot \mathrm{min}^{-1}\right)$. That explains the observed higher mean velocities and justifies a smaller criterion for identifying responders. 
From Neal et al. and the current study, between 31 and $46 \%$ of runners may select a higher velocity following acute caffeine consumption. No data were collected regarding habitual caffeine use of current participants. With previous research failing to definitively determine the potential impact of prior caffeine use on exercise responses, this is mentioned among the factors responsible for inter-individual variability. Reasons for deviant responses among individuals is unclear based on current literature but is worthy of future inquiry.

Three investigations have examined the effects of caffeine on stationary cycling using RPE production. Cole et al. (1996) showed no significant differences at specifically prescribed RPE's of 9, 12 and 15 , but significantly greater cumulative work following caffeine (vs. placebo) which indicates, albeit less convincingly, a potential caffeine effect. As in the current study, Cole et al. (1996) assessed individual responses noting 7 of 10 were positive responders with 3 non-responders (no negative responders). From our lab, Langford et al. (unpublished observation) showed greater workload selection (at a clamped cadence) during cycling at RPE 4 and 7. Also from our lab, Green, et al. (unpublished observation) showed significantly greater mean cadence selection (rev•min-1) at a clamped resistance for RPE4 (CAF $=71 \mathrm{vs}$. PLA $=67)$ but no significant difference at RPE7 $(\mathrm{CAF}=71$, PLA $=70)$. In that study a 'least significant difference' was calculated to be $>5 \mathrm{rev} \bullet \mathrm{min}^{-1}$. Using that criteria, at RPE4, $33 \%$ of observations $(\mathrm{n}=5)$ were positive responses while only 3 (10\%) were at RPE7. Increased VEL from caffeine during RPE production is consistent with Langford et al. (unpublished observation) for RPE4 and RPE7 and with Green et al. (unpublished observation) for lower (RPE4) but not higher (RPE7) intensity. Current results show six positive responses for RPE 4 and four for RPE 7. This study suggests the effects of caffeine may be less pronounced at higher workloads during RPE production. This seems counterintuitive assuming analgesic properties of caffeine are the mechanism for enhanced performance in the context of perceptual responses. More work is needed to fully understand the influence of intensity in the current paradigm. Principle outcomes in the current investigation show mild increases overall in VEL and physiological responses coupled with that change.

$\mathrm{VO}_{2}, \mathrm{HR}$ Response and RER

Results for HR (CAF vs. PLA) are in Figures 1 and 2. With the exception of HR at RPE 4 (Figure 1), mean values for CAF are consistently elevated (vs. PLA) though the magnitude of differences was small 
and statistical significance was rare. As discussed, large standard deviations are evident and highly dispersed data reduce likelihood of significant mean differences. That alone should not discredit effects identified in individual responders. In the current study, the positive responses (10 of 32 trials) averaged 12 $\mathrm{b} \bullet \mathrm{min}^{-1}$ higher for HR for CAF. For overall mean differences (not limiting to responders only) Cole et al. (1996) observed $5 \mathrm{~b} \cdot \mathrm{min}^{-1}$ higher for caffeine while across multiple time points Langford et al. (unpublished observation) showed 5-12 b• $\mathrm{min}^{-1}$ for RPE4 and 11-14 b• $\mathrm{min}^{-1}$ for RPE7.

Mean differences for $\mathrm{VO}_{2}$ in the current study were not significant $(\mathrm{p}=0.17)$ for RPE4 but approached significance for RPE7 $(\mathrm{p}=0.07)$. Follow up tests (RPE7 only) showed significantly higher $\mathrm{VO}_{2}$ for $\mathrm{CAF}$ at minute 6 with differences approaching significance at minute $8(\mathrm{p}=0.08)$ and minute $10(\mathrm{p}=$ 0.06) (Figure 4). Assessing responders only (RPE4 and RPE7), mean $\mathrm{VO}_{2}$ was $5.7 \mathrm{ml} \cdot \mathrm{kg}^{-1} \cdot \mathrm{min}^{-1}$ greater for CAF. Cole et al. (1996) showed overall significantly greater $\mathrm{VO}_{2}$ for CAF $\left(2.23 \mathrm{~L} \mathrm{~min}^{-1}\right)$ vs. PLA (2.01 $\mathrm{L} \cdot \mathrm{min}^{-1}$ ) while Langford et al. reported $5-6 \mathrm{ml} \cdot \mathrm{kg}^{-1} \cdot \mathrm{min}^{-1}$ for CAF across 20 min cycling bouts, which corresponds to current results for responders. For $\mathrm{HR}$ and $\mathrm{VO}_{2}$ values observed for responders agree with previous studies and reflect a practical and meaningful difference attributed to caffeine albeit limited to a select portion of the sample. Though physiological responses were elevated, assessing the means for the entire group may diminish the true effect observed in participants responding favorably. A key finding is that CAF shows an effect even in a paradigm where, statistically, it is unlikely to identify a significant difference due to presence of non-responders.

Enhanced free fatty acid (FFA) mobilization and subsequent use as a metabolic substrate is a proposed ergogenic mechanism of caffeine (Graham 2001, Costill et al.1978). Though the literature in this regard is equivocal (Graham 2001), conceptually, magnified FFA use reduces reliance on limited glycogen stores delaying glycogen depletion, potentially extending time to exhaustion. While the current study did not involve work to exhaustion, respiratory exchange ratio (RER) at sub-maximal workloads may shed light on caffeine effects. Lower RER reflects enhanced FFA use. However, stable (or depressed) RER at higher VEL would also indicate greater FFA relative use. Table 2 shows RER for RPE4 and RPE7 (CAF vs. PLA). P values show no significant difference for CAF vs. PLA for RPE $4(p=0.28)$ even though differences for mean VEL approached significance. Further for RPE7, where VEL was significantly faster for CAF, RER was not significantly different although it approached significance $(p=0.09)$ with lower 
RER for CAF. Stable (or lower) RER at higher VEL in the current study indicates enhanced FFA contribution. However, evaluating RER between trials using only an aggregate analysis is incomplete. Accurate interpretation of RER hinges on acute response to caffeine. Ten trials identified as positive responses (faster VEL for CAF) showed a mean RER change of -0.021 . Seven responders (of 10) showed a decrease or no change in RER for CAF. The remaining three experienced increased RER (0.02 to 0.03$)$. For non-responders (no meaningful change in VEL) (19 trials) mean change for RER was +0.007 . Interpreting RER with regard to CAF response suggests greater FFA use based on blunted or stable RER at elevated VEL. However, for non-responders, current results for RER indicate no meaningful effect on FFA use coupled with no effect for VEL. Therefore, the capacity of caffeine to alter substrate utilization appears limited to positive responders which would be anticipated. Participants showing no meaningful change in VEL showed little change in RER which is also expected. Mechanistically caffeine may alter RPE and enhance FFA mobilization yet evidence in particular for the latter is equivocal (Graham 2001). Current results indicate these responses are coupled or not experienced at all. While plausible, this deserves more attention with direct measures reflecting substrate contribution concurrent with perceptually-linked responses.

Pre-workout energy drinks often contain caffeine. A principle finding of the current study is that caffeine alters perceptually prescribed intensity regulation with a portion of individuals who appear sensitive to caffeine experiencing meaningful ergogenic benefits when consuming caffeine prior to exercise. The practical application from the current study is that pre-exercise caffeine consumption may alter perceptual responses associated with pending exercise. This is important for individual and practitioners when developing training programs and effectively regulating exercise intensity. It is important to be aware of pre-workout nutritional habits and potential impacts on perceived exertion and intensity selection. For positive responders, adjustments may be warranted to achieve desired intensity.

This study was limited to acute responses to caffeine during submaximal constant workload exercise. Assuming responses to caffeine remained consistent, daily training for responders would potentially occur at a systematically higher workload reflected by VEL, HR and $\mathrm{VO}_{2}$. Longitudinally, concordant changes might be expected for fitness. Further, if duration is maintained, higher workloads could result in greater total work volume and caloric expenditure with potential implications to weight 
management efforts. It is emphasized that direct data supporting these hypotheses are lacking and while plausible, they are speculative and should be interpreted as such. Future studies are warranted on the consistency of acute responses to caffeine and potential long-term outcomes. Additional work is warranted also with regard to the potential influence of fitness level or volume of exercise experience. As individuals acquire greater and greater exercise volume, fitness would of course be expected to improve. Further, it would be anticipated that the experience would result in greater precision in regulating exercise intensity which could play a role in the effects observed due to caffeine ingestion. Similarly, future studies should seek to elucidate the potential influence of habitual caffeine use of participants on exercise outcomes.

Caffeine is a widely-investigated ergogenic aid and common ingredient in pre-workout beverages. It is noteworthy that scientific studies most often evaluate effects of caffeine using a dose relative to the body weight of individual participants. This deviates from consumption of energy drinks which contain an absolute amount of caffeine. Individuals and trainers opting to consume caffeine prior to exercise should consider caffeine content and body weight to assess the relative dose being consumed. Caffeine tends to blunt estimated RPE, however, few studies have investigated the influence during RPE production. Although analyses of mean data in the current study reflect a marginal effect, the most thorough and appropriate assessment for caffeine requires evaluation of individual responses. Based on a change in selected treadmill VEL of $13.4 \mathrm{~m} \cdot \mathrm{min}^{-1}\left(0.5 \mathrm{~m} \bullet \mathrm{hr}^{-1}\right)$ during RPE production, $31 \%$ of trials were labeled positive responses. Systematic changes in $\mathrm{HR}$ and $\mathrm{VO}_{2}$ also indicated meaningful effects in responders. Analysis of RER validated that responders not only selected higher VEL but also experienced a positive augmentation of FFA as a substrate. Principle conclusions from the current study correspond with studies indicating high inter-individual variability with regard to caffeine use with a meaningful effect in some. Further work is needed to elucidate specific factors responsible for diverse responses among individuals to acute caffeine supplementation.

Conflict of Interest Statement: The authors declare there are no conflicts of interest. 
Acknowledgments: Authors extend appreciation to all participants as well as support received from Western Kentucky University School of KRS and University of North Alabama Department of HPER for support of the current project. 


\section{References}

American College of Sports Medicine, Guidelines for Exercise Testing and Prescription, $9^{\text {th }}$ ed. 2014, Baltimore, MD, Williams and Wilkins.

Birnbaum, L.J., Herbst, J.D. 2004. Physiologic effects of caffeine on cross-country runners. J. Strength. Cond. Res. 18:463-465.

Cohen, J. Statistical power analysis for the behavioral sciences, $2^{\text {nd }}$ edition, 1988, Hillsdale, NJ: Lawrence Earlbaum Associates

Cole, K.J., Costill, D.L., Starlin, R.D., Goodpaster, B.H., Trappe, S.W., Fink, W.J. 1996. Effect of caffeine ingestion on perception of effort and subsequent work. Int. J. Sports. Nutr.6:14-23.

Costill, D.L., Daskv, G.P, Fink, W.J. 1978. Effects of caffeine on metabolism and exercise performance. Med Sci Sports Exerc. 10: 155-158.

Davis, J.K. and Green, J.M. 2009. Caffeine and anaerobic performance: ergogenic value and mechanisms of action. Sports Med.10: 813 - 832 .

Desbrow, B., Biddulph, C., Devlin, B., Grant, G.D., Anoopkumar-Dukie, S., Leveritt, M.D. 2102. The effects of different doses of caffeine on endurance cycling time trial performance. J. Sports Sci. 30:115120 .

Fredholm, B.B., Bättig, K., Holmén, J., Nehlig, A., Zvartau, E.E. 1999. Actions of caffeine in the brain with special reference to factors that contribute to its widespread use. Pharmacol. Rev. 51: 83-133.

Graham, T.E. 2001. Caffeine and exercise: metabolism, endurance and performance. Sports Med. 31: 785 807.

Jones, A.M. and Doust, J.H. 1996. A 1\% grade most accurately reflects the energetic cost of outdoor running. J. Sports Sci.14: $321-327$.

Killen, L.G., Green, J.M., O’Neal, E.K., McIntosh, J.R., Hornsby, J., Coates, T.E. 2013. Effects of caffeine on session ratings of perceived exertion. Eur. J. Appl. Physiol.113: 721-727.

Laurence, G., Wallman, K., and Guelfi, K. 2012. Effects of caffeine on time trial performance in sedentary men. J. Sports Sci.30:1235-1240. 
Pollock, M.L., Schmidt, D.H. and Jackson, A.S. 1980. Measurement of cardiorespiratory fitness and body composition in the clinical setting. Clinical Therapy. 6:12-27.

Robertson, R.J. and Noble, B.J. 1997. Perception of physical exertion: methods, mediators, and applications. Exerc. Sports Sci. Rev. 25: 407-452.

Sawynok, J., and Yaksh, T.L. 1993. Caffeine as an analgesic adjuvant: A review of pharmacology and mechanisms of action. Pharmacol. Rev. 45: 43-85.

Tallis, J., Duncan, MJ, James, R.S. 2015. What can isolated skeletal muscle experiments tell us about the effects of caffeine on exercise performance? British J Pharm. 172: 3703-3713.

Utter, A.C., Robertson, R.J., Green, J.M., Suminski, R.R., McAnulty, S.R., Nieman, D.C. 2004. Validation of the Adult OMNI Scale of Perceived Exertion for Walking/Running Exercise. Med. Sci. Sports Exerc. 36:1776-1780.

Zhang, W. 2001. A benefit-risk assessment of caffeine as an analgesic adjuvant. Drug Safety. 24:11271142 . 
Table 1. VEL $\left(\mathrm{m} \bullet \mathrm{min}^{-1}\right)(\mathrm{CAF}$ vs. PLA) for RPE4 and RPE7. Values are means \pm std dev. P values (1 tailed) are provided for main effects (means) and follow up analyses at each time point.

\begin{tabular}{llcccccc}
\hline & min & $\mathbf{2}$ & $\mathbf{4}$ & $\mathbf{6}$ & $\mathbf{8}$ & $\mathbf{1 0}$ & mean \\
\hline \multirow{2}{*}{ RPE4 } & CAF & $130 \pm 35$ & $130 \pm 35$ & $132 \pm 36$ & $132 \pm 36$ & $132 \pm 37$ & $131 \pm 35$ \\
& PLA & $124 \pm 29$ & $125 \pm 29$ & $126 \pm 30$ & $126 \pm 30$ & $126 \pm 30$ & $125 \pm 29$ \\
& $\mathrm{p}$ & 0.06 & 0.07 & 0.07 & 0.07 & 0.08 & 0.07 \\
& & & & & & & \\
\multirow{2}{*}{ RPE7 } & CAF & $160 \pm 33$ & $162 \pm 36$ & $163 \pm 37$ & $163 \pm 39$ & $164 \pm 39$ & $162 \pm 36$ \\
& PLA & $152 \pm 28$ & $155 \pm 30$ & $158 \pm 33$ & $160 \pm 35$ & $160 \pm 35$ & $157 \pm 31$ \\
& p & 0.01 & 0.02 & 0.05 & 0.15 & 0.09 & 0.03 \\
\hline
\end{tabular}


Table 2. RER (CAF vs. PLA) for RPE4 and RPE7. Values are means \pm std dev. P values (1 tailed) are provided for main effects (means) and follow up analyses at each time point.

\begin{tabular}{|c|c|c|c|c|c|c|c|}
\hline & $\min$ & 2 & 4 & 6 & 8 & 10 & mean \\
\hline \multirow{3}{*}{ RPE4 } & CAF & $0.90 \pm 0.09$ & $0.93 \pm 0.06$ & $0.94 \pm 0.05$ & $0.93 \pm 0.05$ & $0.93 \pm 0.04$ & $0.93 \pm 0.01$ \\
\hline & PLA & $0.90 \pm 0.08$ & $0.92 \pm 0.07$ & $0.92 \pm 0.06$ & $0.93 \pm 0.05$ & $0.94 \pm 0.06$ & $0.92 \pm 0.02$ \\
\hline & $\mathrm{p}$ & 0.46 & 0.20 & 0.04 & 0.45 & 0.40 & 0.28 \\
\hline \multirow{3}{*}{ RPE7 } & CAF & $0.92 \pm 0.07$ & $0.97 \pm 0.05$ & $0.98 \pm 0.04$ & $0.97 \pm 0.04$ & $0.98 \pm 0.05$ & $0.97 \pm 0.01$ \\
\hline & PLA & $0.91 \pm 0.08$ & $0.95 \pm 0.06$ & $0.96 \pm 0.06$ & $0.96 \pm 0.06$ & $0.97 \pm 0.06$ & $0.95 \pm 0.01$ \\
\hline & $\mathrm{p}$ & 0.24 & 0.04 & 0.06 & 0.17 & 0.19 & 0.09 \\
\hline
\end{tabular}


Figure Captions:

Figure 1. HR (CAF vs. PLA) for RPE4. Values are means \pm standard deviations.

Figure 2. HR (CAF vs. PLA) for RPE7. Values are means \pm standard deviations.

Figure 3. $\mathrm{VO}_{2}(\mathrm{CAF}$ vs. PLA) for RPE4. Values are means \pm standard deviations.

Figure 4. $\mathrm{VO}_{2}$ (CAF vs. PLA) for RPE7. Values are means \pm standard deviations. P values (1 tail) reflect follow up t-tests. 
Figure 1:

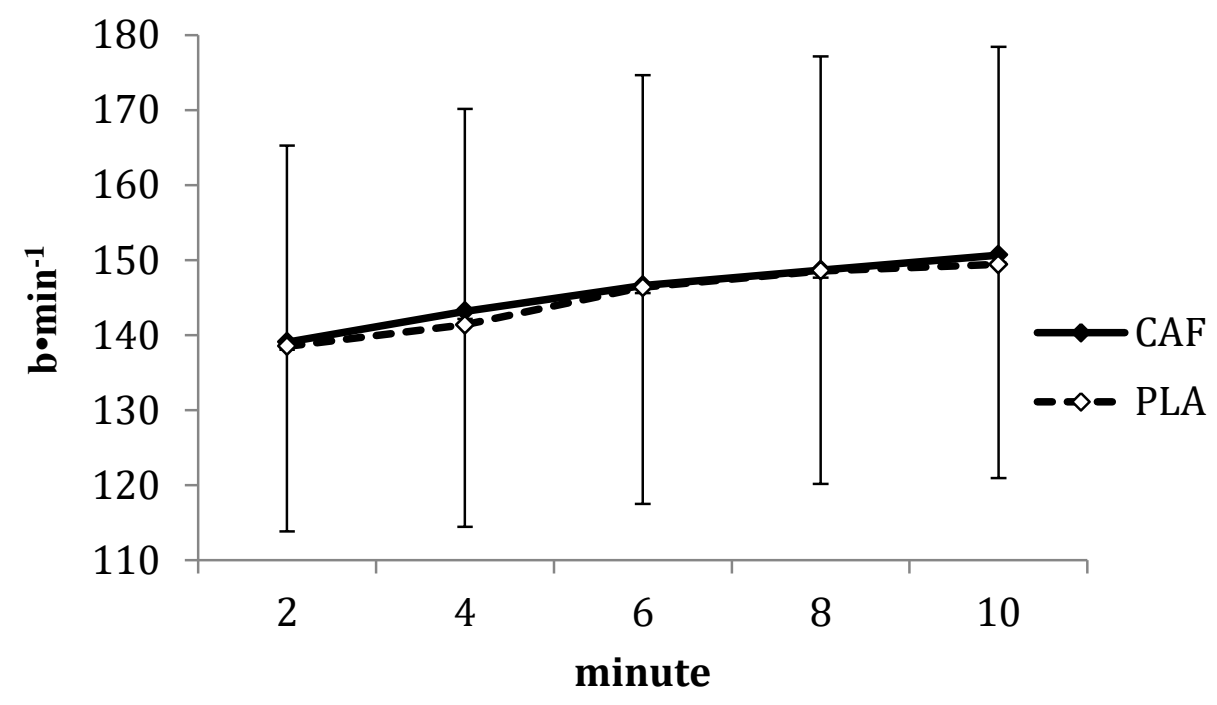


Figure 2:

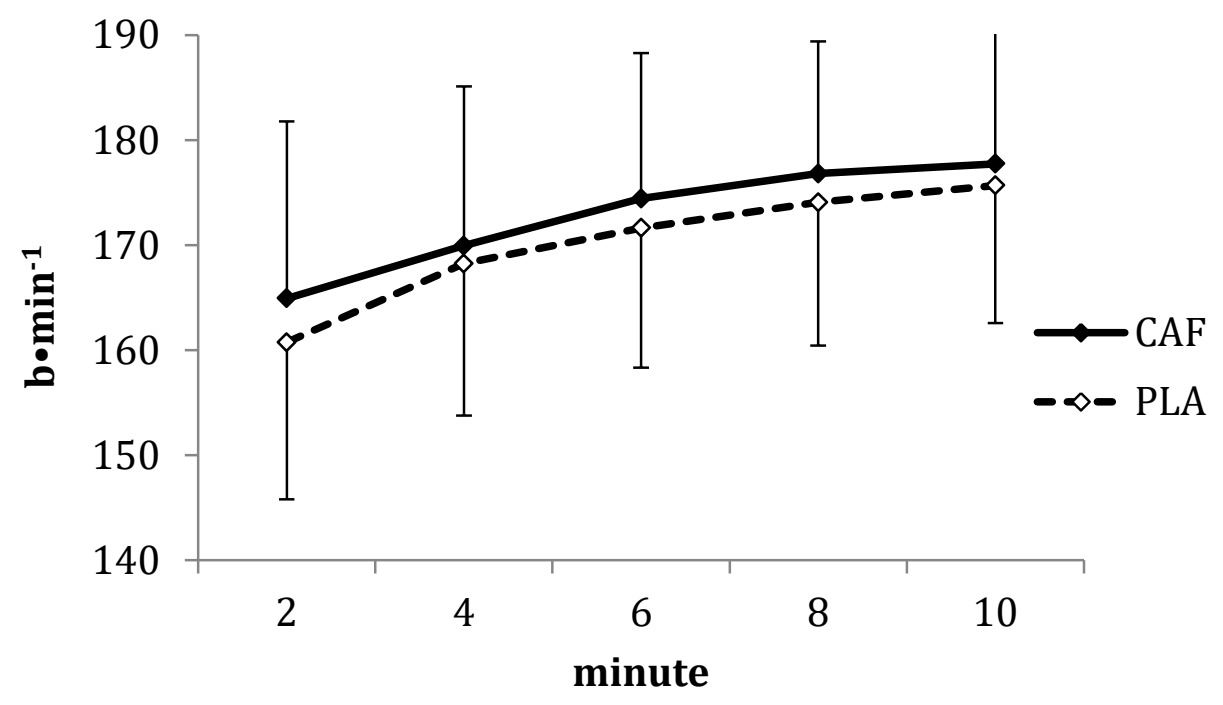


Figure 3:

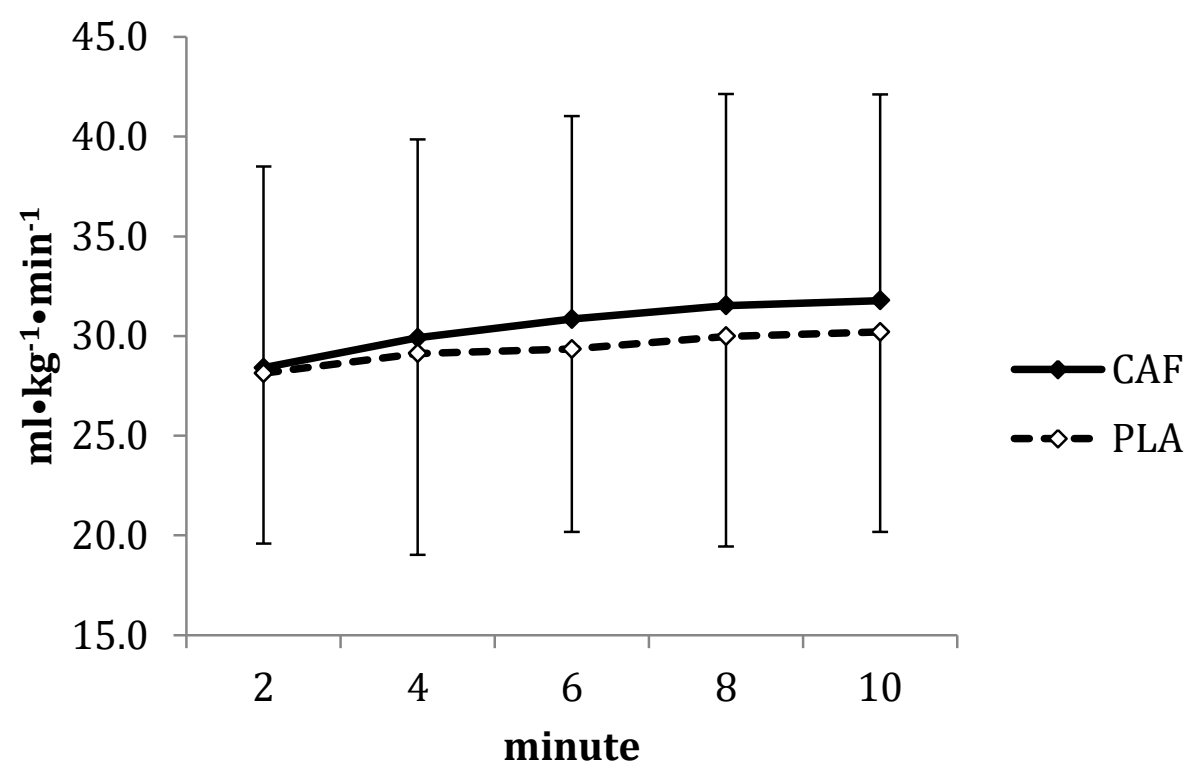


Figure 4:

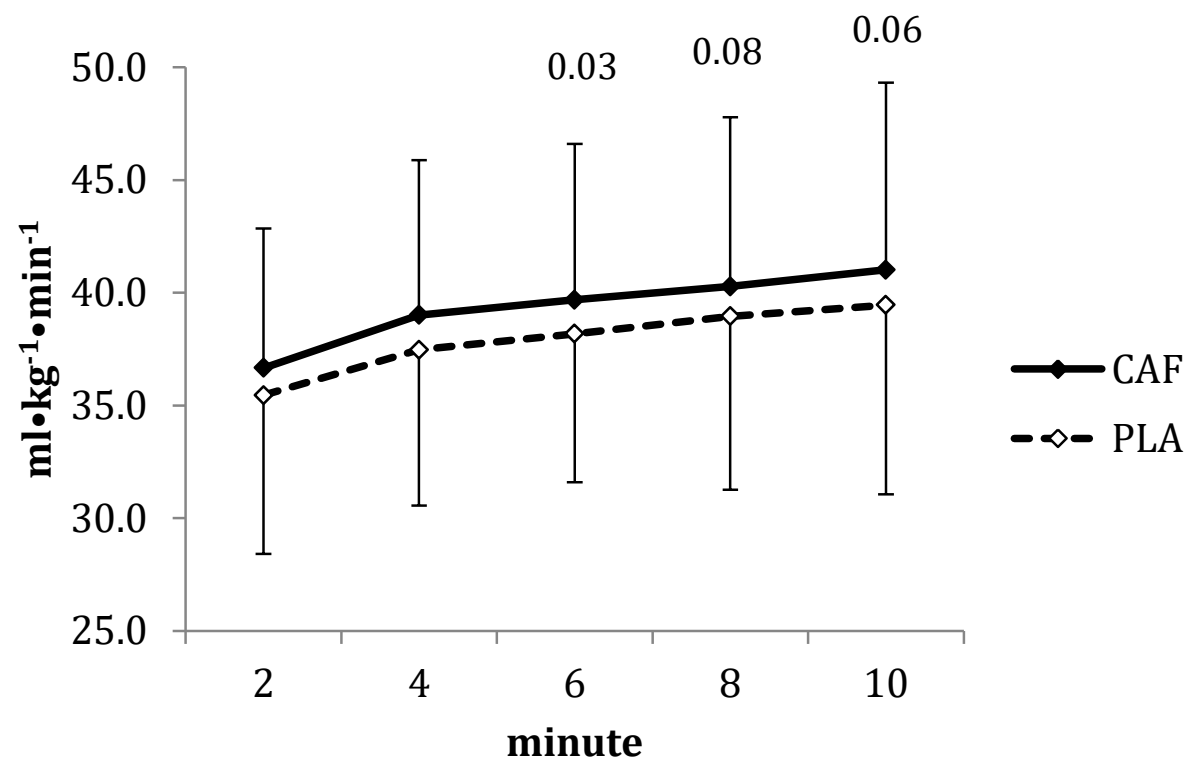

